\title{
Discordance between perception and treatment practices associated with intensive care unit-acquired bacteriuria and funguria: A Canadian physician survey*
}

\author{
Clarence Chant, PharmD, BCPS; Claudia C. Dos Santos, MD; Paola Saccucci, PharmD; \\ Orla M. Smith, RN, MN, CNCC(C); John C. Marshall, MD, FRCS(C); Jan 0. Friedrich, MD, DPhil, FRCP(C)
}

Objective: To determine physician practice and perception about the management of intensive care unit (ICU)-acquired bacteriuria and funguria.

Design: Cross-sectional, self-administered, Web-based survey.

Setting: All provinces within Canada.

Participants: Staff ICU physicians who are members of either the Canadian Critical Care Trials Group or the Canadian Critical Care Society.

Interventions: Survey items were developed by four ICU clinicians, and survey sensibility was assessed by five independent intensivists. Nonrespondents received three follow-up reminders. Participants were asked questions about general perceptions and practices regarding the management of ICU-acquired bacteriuria and funguria. Clinical scenarios were used to elicit management strategies, including antimicrobial prescriptions.

Measurements and Main Results: Ninety of 198 physicians (45\%) responded. Bacteriuria was perceived by $63 \%$ of the respondents to be a frequent but low-morbidity problem. Most intensivists (98\%) did not use a protocol for management. Traditional symptoms were rarely used to interpret the significance of bacteriuria. Presence of systemic inflammatory response syndrome (93\%), presence of hemodynamic changes (91\%), and urinalysis (69\%) were used often. Within clinical scenarios, source control via urinary catheter change was not universal, ranging from $44 \%$ to $67 \%$ in the various scenarios, even in patients presenting with septic shock. Prescription of antimicrobials was common across scenarios despite the low-morbidity perception. In an asymptomatic patient, $19 \%$ of respondents would prescribe antimicrobials. Changing the species from fungus to bacteria and the presence of systemic inflammatory response syndrome or shock increased the likelihood of antimicrobial use up to $70 \%$ to $80 \%$.

Conclusions: ICU physicians perceive bacteriuria to have low morbidity. However, management approaches vary considerably, and systemic antimicrobials are frequently prescribed. Increased clinical instability and bacterial vs. fungal organisms isolated in urine cultures increased the use of antimicrobials. The considerable variability in practice and discordance between likelihood of urinary tract infection and antimicrobial prescription, highlights the need for additional clinical trials. (Crit Care Med 2008; 36:1158-1167)

KeY WoRDS: survey; urinary tract infection; bacteriuria; antibiotic; critical care

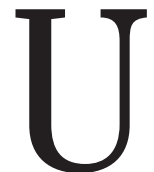

rinary tract infections (UTIs) are one of the most common nosocomial infections within intensive care units (ICUs) in North America (1). The Centers for Disease Control and Prevention categorizes positive urine cultures as either asymptomatic bacteriuria or UTI (2). This definition, however, may not be relevant to

\footnotetext{
*See also p. 1374.

From the Pharmacy Department (CC, PS) and Critical Care Department (CCDS, OMS, JCM, JOF); St. Michael's Hospital; the Interdepartmental Division of Critical Care (CCDS, JCM, JOF); and Leslie Dan Faculty of Pharmacy (CC), University of Toronto; Li Ka Shing Knowledge Translation Institute (JOF), Toronto, Ontario, Canada.

Dr. Friedrich is supported by a Canadian Institutes of Health Research (CIHR) Clinician Scientist Award. This project received no specific funding.
}

ICU-acquired UTIs that are predominantly associated with indwelling urinary catheters and are frequently asymptomatic. It has been estimated that there is a risk of $5 \%$ per day of developing asymptomatic bacteriuria for each day of catheterization (3). Risk factors for the development of UTI in catheterized ICU patients include age $>60 \mathrm{yrs}$, female gen-

Dr. Marshall has consulted for Becton Dickinson, Takeda, and Pfizer and has received honoraria/speaking fees from Spectral Diagnostics. The remaining authors have not disclosed any potential conflicts of interest.

For information regarding this article, E-mail: chantc@smh.toronto.on.ca

Copyright $\odot 2008$ by the Society of Critical Care Medicine and Lippincott Williams \& Wilkins

DOI: 10.1097/CCM.0b013e3181692af9 der, length of ICU stay, prior use of antibiotics, severity of illness, and duration of catheterization (4-6). UTIs are associated with increased hospital costs and prolonged hospitalizations, despite the fact that they are not associated with a high incidence of bacteremia and/or fungemia (7). Whether UTIs lead to a significant increase in attributable mortality in critically ill patients remains controversial. Adjusting for confounders, studies in the critically ill have generally not documented an increase in mortality in patients with UTI, although recent data suggest that candiduria may be associated with an increased rate of hospital mortality $(7,8)$.

The diagnosis of ICU-acquired UTI is problematic due to the paucity of literature as well as the lack of reliability and specificity of clinical signs and symptoms in critically ill patients. Leone et al. (9) 
systematically reviewed the literature on catheter-associated UTI and found 24 clinical trials, of which only eight involved ICU patients and none were randomized control trials. Given this lack of high-level evidence, clinical decisions about ICU-acquired bacteriuria or funguria are based on local practice patterns and personal experiences, leading to the potential for significant variations in practice. These variations may lead to either over- or undertreatment with antimicrobials and so affect resistance rates, costs, and adverse patient outcomes. We conducted a Webbased survey to assess physician practices and perceptions related to the management of positive urine cultures in critically ill patients in Canadian ICUs.

\section{MATERIALS AND METHODS}

Instrument Development. The survey was developed by an interprofessional team of critical care clinicians (two physicians, one nurse, and one pharmacist). All questions were closeended and all frequency type questions used a 5 -point Likert scale recorded as "almost every time," "often," "sometimes," "rarely," and "almost never." The draft survey was pretested by five intensivists for clarity and for face and content validity. Modifications were made based on the feedback.

The survey (Appendix A) was divided into three sections, consisting of clinical scenarios, general perceptions, and demographics. The scenarios were designed as follows: The base case (scenario 1) described an ICU patient with asymptomatic bacteriuria with an organism typically causing UTI (Escherichia coli); scenario $2 \mathrm{~A}$ described the same patient with bacterial species and symptoms of systemic inflammatory response syndrome (SIRS); scenarios $2 \mathrm{~B}$ and $2 \mathrm{C}$ described two additional variations using organisms less typically associated with symptomatic bacteriuria, Enterococcus faecalis (2B) and Candida albicans (2C); scenario 3 described the same patient with $E$. coli bacteriuria in addition to a concurrent pneumonia caused by methicillinresistant Staphylococcus aureus (MRSA) that could potentially account for the SIRS symptoms; and the final scenario (scenario 4) described the same patient with a urine culture positive for an organism infrequently associated with symptomatic urinary tract infection (C. albicans) accompanied by septic shock with no positive bacterial source in the cultures. For each scenario, the respondents were asked to indicate their perception of the probability that the patient had a true UTI and their management strategies (Fig. 1).

Instrument Administration. With permission from the respective chairs, the membership contact lists of both the Canadian Critical Care Trials Group and the Canadian Critical Care Society were combined to identify poten-

2A: ICU-acquired bacteriuria with E. coli and SIRS

2B: ICU-acquired bacteriuria with Enterococcus faecalis and SIRS

2C: ICU-acquired bacteriuria with Candida albicans and SIRS

3: $\quad$ ICU-acquired bacteriuria with E. coli and MRSA ventilator-associated pneumonia

4: $\quad$ ICU-acquired bacteriuria with $C$. albicans and shock

Each scenario with the following options:

$\square$ No specific treatment required for the E.coli

$\square$ Repeat urine culture

Change foley catheter

Start systemic antibiotics for the E.coli

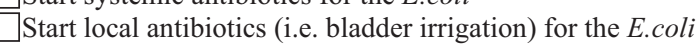

$\checkmark$ Start systemic antibiotics based on repeat culture results

$\checkmark$ Start local antibiotics (i.e. bladder irrigation) based on repeat culture results

$\square$ Other. Please specify:

In your view, how likely is this woman to have a urinary tract infection (UTI)?
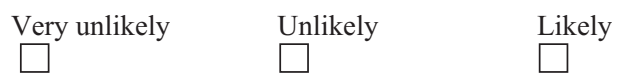

Very Likely

Figure 1. Clinical scenarios used in the survey. ICU, intensive care unit; SIRS, systemic inflammatory response syndrome; MRSA, methicillin-resistant Staphylococcus aureus; UTI, urinary tract infection.

tial participants. Duplicate names and physicians not actively practicing in an adult ICU in Canada were removed. The remaining physicians were invited to complete the survey online (www.surveymonkey.com, Portland, OR). Two automatic reminder e-mails were sent at monthly intervals followed by a final personalized reminder. Internet searches and personal contacts were used in an attempt to correct undeliverable e-mail addresses. The protocol was approved by the Research Ethics Board of our institution.

Analysis. All returned surveys with at least one complete answer were included in the analysis. Data are reported as mean $( \pm \mathrm{SD})$ or median (interquartile range) as appropriate. Where appropriate, responses were compared using Fisher's exact test using SAS software version 8.2 (SAS Institute, Cary, NC).

\section{RESULTS}

After removal of duplicate names, the combined membership lists yielded a pool of 198 potential respondents. Ninety surveys were returned, for a response rate of $45 \%$. Most respondents had postgraduate training in critical care, and their current practice involved treating a variety of critically ill patients in mediumsized (10-20 beds) closed ICUs in university-affiliated institutions (Table 1).

Responses to General Perception Questions. Most respondents perceived healthcare-associated positive urine cultures to occur frequently $(63 \%)$ in the ICU. The majority (98\%) of respondents did not use a standardized protocol to manage ICUacquired bacteriuria. Frequency of use of various diagnostic variables is summarized in Table 2. Many physicians would use urine microscopy (69\%) and biochemistry (50\%) almost every time or often, while the majority would use clinical indicators of severity of illness, such as SIRS (93\%), or hemodynamic status (91\%) almost every time or often to guide management. In contrast, traditional UTI symptoms were rarely or almost never (60\%) used.

General management approaches also demonstrated wide variability, with respondents frequently changing the urinary catheters, either alone or in combination with repeat cultures $(67 \%)$. Respondents tended to be less certain regarding systemic antimicrobials, with the majority (75\%) reporting that they either sometimes or frequently prescribed them. However, the majority of the respondents $(93 \%)$ believed that positive cultures only sometimes or rarely led to bacteremia/fungemia, and rarely or almost never $(85 \%)$ led to death.

Response to Clinical Scenarios. Figure 2 shows the proportion of respondents who felt that the patient was likely or very likely to have an ICUacquired UTI along with the proportion who would prescribe systemic antimicrobials and change the urinary catheter for each scenario. Overall, use of local antimicrobials (e.g., bladder irri- 
Table 1. Demographic information of the respondents

\begin{tabular}{|c|c|}
\hline Variable & No. $(\%)$ of Respondents $(\mathrm{n}=84)^{a}$ \\
\hline \multicolumn{2}{|l|}{ Location of practice } \\
\hline Western provinces & $23(27)$ \\
\hline Ontario & $42(50)$ \\
\hline Quebec & $14(17)$ \\
\hline Atlantic provinces & $5(6)$ \\
\hline \multicolumn{2}{|l|}{ Hospital type } \\
\hline University & $63(75)$ \\
\hline Community & $9(11)$ \\
\hline Community with university affiliation & $12(14)$ \\
\hline \multicolumn{2}{|l|}{ Type of ICU } \\
\hline Closed & $79(94)$ \\
\hline Open & $5(6)$ \\
\hline \multicolumn{2}{|l|}{ ICU bed capacity } \\
\hline$<10$ & $3(4)$ \\
\hline $11-20$ & $46(53)$ \\
\hline $21-30$ & $28(33)$ \\
\hline$>30$ & $9(11)$ \\
\hline \multicolumn{2}{|l|}{ Postgraduate training in critical care } \\
\hline Yes & $75(89)$ \\
\hline No & $9(11)$ \\
\hline \multicolumn{2}{|l|}{ Years of ICU experience } \\
\hline$<5$ & $23(27)$ \\
\hline $6-15$ & $39(46)$ \\
\hline $16-25$ & $17(20)$ \\
\hline$>25$ & $5(6)$ \\
\hline \multicolumn{2}{|l|}{ Patient population (more than one applies) } \\
\hline Surgical & $78(93)$ \\
\hline Medical & $75(89)$ \\
\hline Burn & $20(24)$ \\
\hline Trauma & $37(44)$ \\
\hline Coronary care & $9(11)$ \\
\hline Neurosurgical & $38(45)$ \\
\hline Respiratory & $50(60)$ \\
\hline Transplant & $21(25)$ \\
\hline Cardiothoracic & $29(35)$ \\
\hline Chronic & $26(31)$ \\
\hline
\end{tabular}

ICU, intensive care unit.

${ }^{a}$ Six surveys were returned without demographic information.

Table 2. Use of diagnostic tests and/or clinical variables in management of intensive care unit-acquired bacteriuria or funguria

\begin{tabular}{|c|c|c|c|c|c|}
\hline & $\begin{array}{c}\text { Almost } \\
\text { Every Time }\end{array}$ & Often & Sometimes & Rarely & $\begin{array}{c}\text { Almost } \\
\text { Never }\end{array}$ \\
\hline Urine microscopy & $40(47)$ & $19(22)$ & $10(12)$ & $8(9)$ & $8(9)$ \\
\hline Urine biochemistry & $25(29)$ & $18(21)$ & $9(11)$ & $20(24)$ & $13(15)$ \\
\hline Urine appearance & $14(16)$ & $26(31)$ & $25(29)$ & $11(13)$ & $9(11)$ \\
\hline Symptoms & $6(7)$ & $15(18)$ & $13(15)$ & $26(31)$ & $25(29)$ \\
\hline SIRS & $50(59)$ & $29(34)$ & $5(6)$ & $1(1)$ & $0(0)$ \\
\hline Hemodynamic changes & $49(58)$ & $28(33)$ & $6(7)$ & $2(2)$ & $0(0)$ \\
\hline
\end{tabular}

SIRS, systemic inflammatory response syndrome.

Values are reported as number of responses (\%).

gation) was low, with fewer than three respondents selecting this method of management for any scenario.

In an asymptomatic and clinically stable ICU patient with $E$. coli bacteriuria, $26 \%$ considered this likely to be a UTI, $49 \%$ would change the urinary catheter, and $19 \%$ would prescribe systemic antimicrobials. Interestingly, of those who chose to prescribe antimicrobials, five of
$17(29 \%)$ considered the patient to be unlikely or very unlikely to have a UTI. Adding the presence of SIRS and no other apparent source of infection to $E$. coli bacteriuria significantly increased the percentage of respondents who considered the patient likely to have a UTI from $26 \%$ to $86 \%(p<.001)$, with a concomitant increase in the prescription of systemic antimicrobials (19\% to $79 \%, p<$
.001) but a similar rate of catheter changes ( $49 \%$ to $44 \%, p=.55$ ).

In E. coli bacteriuria with SIRS and concurrent MRSA pneumonia, $78 \%$ of respondents prescribed antimicrobial therapy for the pneumonia while $53 \%$ also prescribed antimicrobials for the $E$. coli in the urine. This was less than the proportion who prescribed antimicrobials in the presence of SIRS without an alternative source of infection ( $53 \%$ vs. $79 \%, p<.001)$ but greater than in the instance of asymptomatic bacteriuria ( $19 \%$ vs. $53 \%$, $p<.001)$. Of the 46 respondents who prescribed antimicrobials to treat the presumed UTI, only three chose to prescribe antimicrobials strictly for the $E$. coli and not the pneumonia. As before, the proportion of respondents changing the urinary catheter remained unchanged ( $49 \%$ vs. $48 \%, p=1.00)$.

Effect of Different Organisms. In a patient with bacteriuria and SIRS but no other apparent source of infection, changing the organism from $E$. coli to $E$. faecalis resulted in no significant change in the proportion of respondents who considered the patient likely to have a UTI $(86 \%$ vs. $85 \%, p=1.00)$, prescribed systemic antimicrobials $(79 \%$ vs. $76 \%, p=$ $.72)$, or changed the urinary catheter (44\% vs. $47 \%, p=.76$ ). Changing the organism from $E$. coli to $C$. albicans in patients with SIRS, a lower proportion of respondents considered the patient likely to have a UTI $(86 \%$ vs. $24 \%, p<.001)$ and prescribed systemic antimicrobials $79 \%$ vs. $18 \%, p<.001$ ). However, a significantly higher proportion changed the urinary catheter ( $44 \%$ vs. $67 \%, p=.004)$.

Finally, although a relatively low proportion of respondents would treat $C$. albicans in the urine in the presence of SIRS, a substantially higher proportion of respondents would treat with systemic antifungals in the presence of septic shock (18\% vs. $64 \%, p<.001)$, even though only a slightly higher proportion considered the patient likely to have a UTI ( $24 \%$ vs. $40 \%, p=.03)$ and no change in the rate of catheter changes $(67 \%$ vs. $62 \%, p=.63)$.

Regional Variation. Responses were grouped into four geographical regions (Western Canada, Ontario, Quebec, and Atlantic provinces) for post hoc exploratory analysis. There were no differences in general perceptions of the respondents in terms of whether UTI led to bacteremia or death. Within the clinical scenarios, no consistent regional differences were observed. 


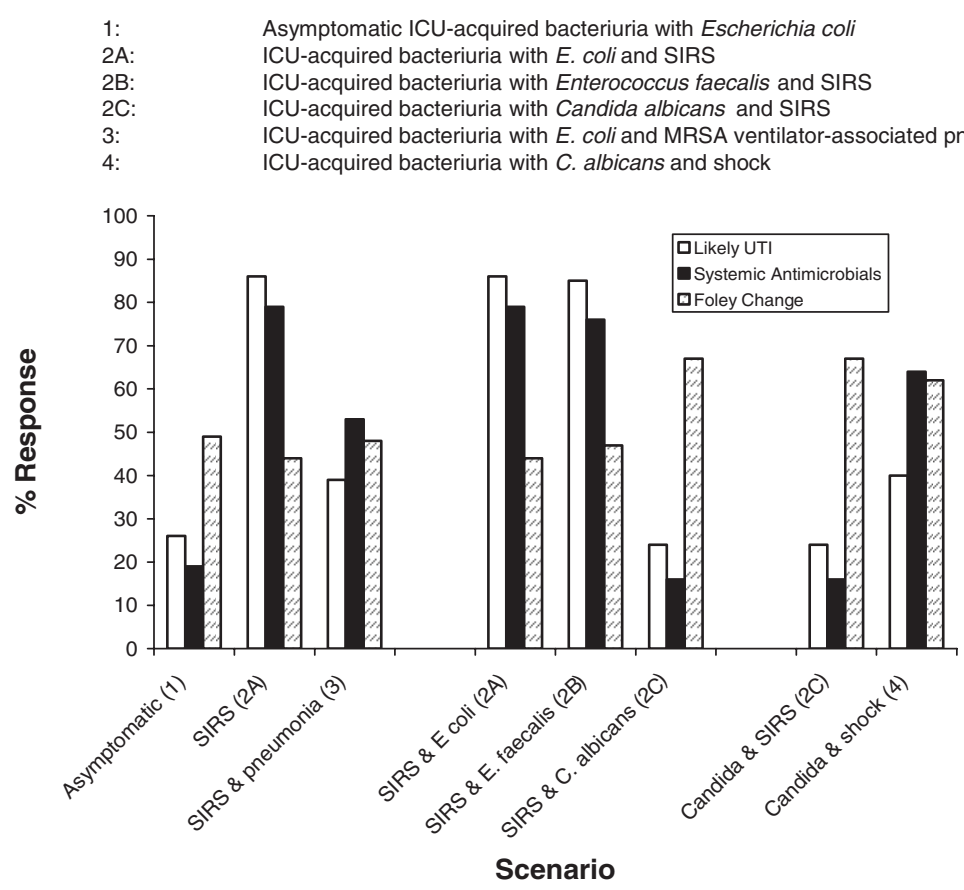

Figure 2. Perceived likelihood of intensive care unit (ICU)-acquired bacteriuria/funguria and management approach. SIRS, systemic inflammatory response syndrome; MRSA, methicillin-resistant Staphylococcus aureus; UTI, urinary tract infection.

\section{DISCUSSION}

The primary finding of this survey is that a significant number of ICU physicians would prescribe systemic antimicrobials for ICU-acquired bacteriuria despite the perception that it is associated with low morbidity and risk of progression to systemic infection, even when the patient was asymptomatic. Some variability in practice is expected given the lack of evidence in this area, as has been demonstrated even in areas with large bodies of literature, such as acute respiratory distress syndrome (10). Standardization of care processes via evidence-based protocols are known to enhance knowledge translation and patient outcomes; therefore, the current survey highlights the importance of further research, especially given the consequences associated with inappropriate antimicrobial prescription (11).

Antimicrobial Prescription. In our survey, the decision to prescribe systemic antimicrobials was most strongly influenced by the clinical stability of the patient, in particular the presence of SIRS or shock. Nineteen percent of our respondents would prescribe systemic antimicrobials for asymptomatic E. coli bacteriuria. This practice contrasts with the findings of previous studies in non-ICU patients as well as another recent randomized controlled trial conducted in the ICU which demonstrated that no specific treatment (i.e., no catheter change and no antimicrobials) resulted in similar patient outcomes as aggressive treatment (i.e., catheter change after first dose of a 3-day course of antimicrobials) (12-15). However, clinical details about the patient population (e.g., asymptomatic bacteriuria vs. UTI) were not provided in the ICU study, and therefore direct comparisons to our hypothetical patient scenarios cannot be made. The randomized study in the ICU had a small sample size of 30 patients in each group; thus the shorter length of stay in the conservative treatment group (19 vs. 28 days) did not reach statistical significance. Nevertheless, this trial suggests that no specific therapy (i.e., no catheter change and no antimicrobials) may be safe and that perhaps the respondents who prescribed antimicrobials to our asymptomatic patient may have done so unnecessarily. Moreover, biofilm formation on catheters is known to predispose toward bacterial or fungal colonization and prevents antimicrobial penetration (16). The resultant implication is that if any treatment is required, catheter change may be more beneficial than antimicrobials.

Urinary Catheter Change. Respondents clearly view funguria to be less pathogenic than bacteriuria, as indicated by the significant decrease in systemic antimicrobial prescription when only the organism in the culture, and not the clinical context, was changed in our scenarios (Fig. 2). The changing of urinary catheters is a minimal risk option for funguria that is supported by published guidelines, especially in cases of C. albicans (17). Similar to the findings of Avenyi et al. (18), our survey respondents did not universally use this management strategy. When presented with a scenario of funguria and concomitant SIRS, only two thirds of respondents said that they would change the urinary catheter. Evidence from randomized trials in a hospitalized (nonICU) population shows that catheter change provides the same long-term fungal eradication as treatment with systemic antifungal therapy $(19,20)$.

Limitations. Our study is limited by a response rate of $45 \%$ and a predominance of respondents from academic institutions. Thus, our findings may not accurately reflect practices and perceptions of all practicing ICU physicians. However, one might speculate that there is a potential for less awareness of evidence-based practices among nonacademic intensivists and thus even greater practice variability. The survey was only distributed in English electronically and thus may have excluded some French-speaking physicians or those who did not have readily available access to the Internet. The low number of respondents in each region meant that the study was underpowered to detect regional differences. In addition, we acknowledge the biases inherent in self-report methodologies of a survey that do not capture the discrepancies between responses to hypothetical scenarios and actual practice behaviors. However, the development of our survey was standardized, was multidisciplinary in nature, and used scenarios that accurately reflect actual patient situations. Moreover, the draft instrument was pretested by five independent intensivists for clarity, face validity, and content validity using a standardized tool. Web-based administration allowed for ease of response and minimized time commitment. The follow-up was also comprehensive, including Internet searches to correct any wrong or missing e-mail addresses and personal communication to encourage responses.

\section{CONCLUSIONS}

In a national survey of adult intensivists, ICU-acquired bacteriuria was perceived to be a common, but nonlethal, disease process. Management approaches varied widely and depended on the species of organism isolated. Clinical stability most fre- 
quently determined the aggressiveness of treatment of ICU-acquired bacteriuria, even for less pathogenic organisms. Antimicrobial prescription was common despite the perception of low risk. This discordance between perceptions and management approaches highlights the need for additional research to optimize the care of patients presenting with ICU-acquired bacteriuria.

\section{REFERENCES}

1. Richards MJ, Edwards JR, Culver DH, et al: Nosocomial infections in combined medical-surgical intensive care units in the United States. Infect Control Hosp Epidemiol 2000; 21:510-515

2. Centers for Disease Control Web site. http://www.cdc.gov/ncidod/dhqp/pdf/nnis/ NosInfDefinitions.pdf. accessed July 11, 2007

3. Saint S, Lipsky BA: Preventing catheterrelated bacteriuria. Arch Intern Med 1999; 159:800-808

4. Laupland KB, Zygun DA, Davies HD, et al: Incidence and risk factors for acquiring nosocomial urinary tract infection in the critically ill. J Crit Care 2002; 17:50-57

5. Leone M, Albanese J, Garnier F, et al: Risk factors of nosocomial catheter-associated urinary tract infection in a polyvalent inten- sive care unit. Intensive Care Med 2003; 29: 1077-1080

6. Rosser CJ, Bare RL, Meredith JW: Urinary tract infections in the critically ill patient with a urinary catheter. Am J Surg 1999; 177:287-290

7. Laupland KB, Bagshaw SM, Gregson DB, et al: Intensive care unit-acquired urinary tract infections in a regional critical care system. Crit Care 2005; 9:R60-R65

8. Alvarez-Lerma F, Nolla-Salas J, Leon C, et al: Candiduria in critically ill patients admitted to intensive care medical units. Intensive Care Med 2003; 29:1069-1076

9. Leone M, Garnier F, Avidan M, et al: Catheter-associated urinary tract infections in intensive care units. Microbe Infect 2004; 4:1026-1032

10. Meade MO, Jacka MJ, Cook DJ, et al: Survey of interventions for the prevention and treatment of acute respiratory distress syndrome. Crit Care Med 2004; 32:946-954

11. Micek ST, Roubinian N, Heuring T, et al: Before-after study of a standardized hospital order set for the management of septic shock Crit Care Med 2006; 34:2702-2713

12. Nicolle LE, Mayhew WJ, Bryan L: Prospective randomized comparison of therapy and no therapy for asymptomatic bacteriuria in institutionalized elderly women. Am J Med 1987; 83:27-33

13. Nicolle LE, Bjornson J, Harding GK, et al:
Bacteruria in elderly institutionalized men. N Engl J Med 1983; 309:1420-1425

14. Abrutyn E, Mossey J, Berlin JA, et al: Does asymptomatic bacteriuria predict mortality and does antimicrobial treatment reduce mortality in elderly ambulatory women? Ann Intern Med 1994; 120:827-833

15. Leone M, Perrin A, Granier P, et al: A randomized trial of catheter change and short course of antibiotics for asymptomatic bacteriuria in catheterized ICU patients. Intensive Care Med 2007; 33:726-729

16. Nickel JC, Gristina AG, Costerton JW: Electron microscopic study of an infected Foley catheter. Can J Surg 1985; 28:50-51, 54

17. Pappas PG, Rex JH, Sobel JC, et al: Guidelines for treatment of candidiasis. Clin Infect Dis 2004; 38:161-189

18. Ayeni O, Riederer KM, Wilson FM, et al: Clinicians' reaction to positive urine for Candida organisms. Mycoses 1999; 42: 285-289

19. Nassoura Z, Ivatury RR, Simon RJ, et al: Candiduria as an early marker of disseminated infection in critically ill surgical patients: The role of fluconazole therapy. J Trauma 1993; 35:290-294

20. Sobel JD, Kauffman CA, McKinsey D, et al: Candiduria: A randomized, double-blind study of treatment with fluconazole and placebo. Clin Infect Dis 2000; 30:19-24 


\section{Part 1: Clinical Vignettes}

\section{Scenario 1}

Mrs. R.S. is a 68 year old female admitted to the intensive care unit secondary to a drug overdose. She was intubated for airway protection secondary to a decreased level of consciousness. A foley catheter was also placed on ICU admission. Her admission chest x-ray was clear and there was no other evidence of aspiration. The rest of her investigations and lab work were all within normal limits

On day 4 of her ICU stay, her vital signs were as follows: Temperatureafebrile $\left(36.5^{\circ} \mathrm{C}\right)$; $\mathrm{HR}-78$ beats per minute; RR:24 per minute on mechanical ventilation; $\mathrm{BP}-130 / 78 \mathrm{mmHg}$. A urine culture was obtained that was positive for $>10^{5}$ colony forming units $(\mathrm{CFU} / \mathrm{mL})$ E. coli. Her white blood cell (WBC) was 8,000 cells per cubic millimeter (cmm) (Norm: 4,300-10,800). All other investigations were normal.

No specific treatment required for Escherichia coli.

Repeat urine culture.

Change Foley catheter.

Start systemic antibiotics for E. coli.

Start local antibiotics (i.e., bladder irrigation) for E. coli.

Start systemic antibiotics based on repeat culture results.

$\square$ Start local antibiotics (i.e., bladder irrigation) based on repeat culture results.

Other. Please specify:

In your view, how likely is this woman to have a urinary tract infection?

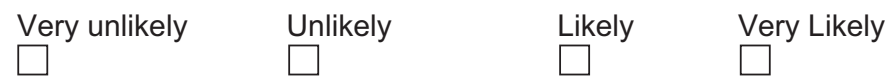

\section{Scenario $2 A$}

Mrs. R.S. is a 68 year old female admitted to the intensive care unit
secondary to a drug overdose. She was intubated for airway protection
secondary to decreased level of consciousness. A foley catheter was also
placed on ICU admission. Her admission chest x-ray was clear and there
was no other evidence of aspiration. The rest of her investigations and lab
work were all within normal limits
On day 4 of her ICU stay, her vitals were as follows: Temperature-
$38.5^{\circ} \mathrm{C} ; \mathrm{HR}-110$ beats per minute; RR: 24 per minute on mechanical
ventilation; BP-130/78 $\mathrm{mmHg}$. A urine culture was obtained that was
positive for $>10^{5} \mathrm{CFU} / \mathrm{mL}$ E.coli. Her white blood cell (WBC) was 15,000
ccm. Her chest x-ray was clear and there was no other source of infection.
and no other source of infection was identified. All other investigations were
normal.

What is your approach for the management of bacteriuria in this patient? (Select all that apply)

No specific treatment required for $E$. coli.

Repeat urine culture.

$\square$ Change Foley catheter.

$\checkmark$ Start systemic antibiotics for E. coli.

Start local antibiotics (i.e., bladder irrigation) for E. coli.

Start systemic antibiotics based on repeat culture results.

Start local antibiotics (i.e., bladder irrigation) based on repeat culture results.

Other. Please specify:

In your view, how likely is this woman to have a urinary tract infection?

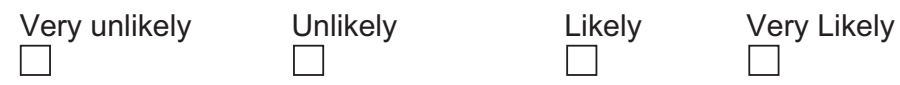


Mrs. R.S. is a 68 year old female admitted to the intensive care unit secondary to a drug overdose. She was intubated for airway protection secondary to decreased level of consciousness. A foley catheter was also placed on ICU admission. Her admission chest $\mathrm{x}$-ray was clear and there was no other evidence of aspiration. The rest of her investigations and lab work were all within normal limits

On day 4 of her ICU stay, her vitals were as follows: Temperature$38.5^{\circ} \mathrm{C}$; HR-110 beats per minute; RR:24 per minute on mechanical ventilation; $\mathrm{BP}-130 / 78 \mathrm{mmHg}$. A urine culture was obtained that was positive for $>10^{5}$ CFU/mL Enterococcus faecalis. Her white blood cell (WBC) was $15 \mathrm{ccm}$. Her chest $\mathrm{x}$-ray was clear and no other source of infection was identified. All other investigations were normal.

What is your approach for the management of bacteriuria in this patient? (Select all that apply)

$\square$ No specific treatment required for Enterococcus faecalis.

$\square$ Repeat urine culture.

Change Foley catheter.

Start systemic antibiotics for E. faecalis.

Start local antibiotics (i.e., bladder irrigation) for $E$. faecalis.

Start systemic antibiotics based on repeat culture results.

Start local antibiotics (i.e., bladder irrigation) based on repeat culture results.

Other. Please specify:

In your view, how likely is this woman to have a urinary tract infection?

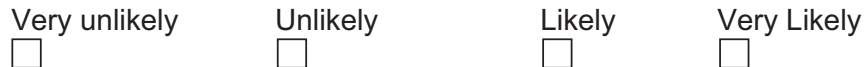

\section{Scenario $2 C$}

Mrs. R.S. is a 68 year old female admitted to the intensive care unit secondary to a drug overdose. She was intubated for airway protection secondary to decreased level of consciousness. A foley catheter was also placed on ICU admission. Her admission chest $\mathrm{x}$-ray was clear and there was no other evidence of aspiration. The rest of her investigations and lab work were all within normal limits.

On day 4 of her ICU stay, her vitals were as follows: Temperature$38.5^{\circ} \mathrm{C}$; HR-110 beats per minute; RR:24 per minute on mechanical ventilation; $\mathrm{BP}-130 / 78 \mathrm{mmHg}$. A urine culture was obtained that was positive for $>10^{5} \mathrm{CFU} / \mathrm{mL}$ Candida albicans. Her white blood cell (WBC) was $15 \mathrm{ccm}$. Her chest $\mathrm{x}$-ray was clear.

All other investigations were normal

What is your approach for the management of bacteriuria in this patient? (Select all that apply)

$\square$ No specific treatment required for $C$. albicans.

Repeat urine culture.

$\square$ Change Foley catheter.

$\square$ Start systemic antifungals for $C$. albicans.

Start local antifungals (i.e., bladder irrigation) for C. albicans.

$\square$ Start systemic antifungals based on repeat culture results.

Start local antifungals (i.e., bladder irrigation) based on repeat culture results.

Other. Please specify:

In your view, how likely is this woman to have a urinary tract infection?

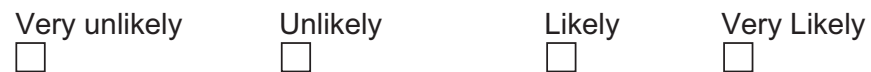


Mrs. R.S. is a 68 year old female admitted to the intensive care unit secondary to a drug overdose. She was intubated for airway protection secondary to decreased level of consciousness. A foley catheter was also placed on ICU admission. Her admission chest x-ray was clear and there was no other evidence of aspiration. The rest of her investigations and lab work were all within normal limits

On day 4 of her ICU stay, her vitals were as follows: Temperature$38.5^{\circ} \mathrm{C}$; HR-110 beats per minute; RR:24 per minute on mechanical ventilation; BP-130/78 $\mathrm{mmHg}$. She had a significant increase in mucopurulent secretions from her endotracheal tube and her chest $\mathrm{x}$-ray showed a new consolidation in the right lower lobe. Sputum culture positive for Staphylococcus aureus MRSA. A urine culture was obtained that was positive for $>10^{5} \mathrm{CFU} / \mathrm{mL}$ E.coli. All other investigations were normal.

What is your approach for the management of bacteriuria in this patient? (Select all that apply)

$\square$ No specific treatment required for Escherichia coli.

Repeat urine culture.

$\square$ Change Foley catheter.

$\square$ Start systemic antibiotics to cover MRSA and E. coli.

Start systemic antibiotics for $E$. coli only.

Start local antibiotics (i.e., bladder irrigation) for E. coli only.

$\square$ Start systemic antibiotics based on repeat culture results.

Start local antibiotics (i.e., bladder irrigation) based on repeat culture results.

Other. Please specify:

In your view, how likely is this woman to have a urinary tract infection?

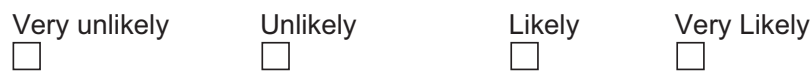

Scenario 4

Mrs. R.S. is a 68 year old female admitted to the intensive care unit secondary to a drug overdose. She was intubated for airway protection secondary to decreased level of consciousness. A foley catheter was also placed on ICU admission. Her admission chest x-ray was clear and there was no other evidence of aspiration. The rest of her investigations and lab work were all within normal limits

On day 4 of her ICU stay, her vitals were as follows: Temperature-39.5 ${ }^{\circ} \mathrm{C}$; HR-135 beats per minute; RR-24 per minute on mechanical ventilation; BP- $-90 / 60 \mathrm{mmHg}$ despite fluid resuscitation. The patient was in vasodilatory shock, presumably due to sepsis. The patient was placed on a norepinephrine infusion requiring higher doses. Urine, blood and sputum specimens were sent for culture and sensitivity. Pending blood culture results, the patient was started empirically on imipenem/cilastatin for broad spectrum coverage and corticosteroids.

Two days later (day 6 ) of her ICU stay the patient remained unchanged and continued to be unstable requiring high doses of inotropes. Repeat urine, blood and sputum specimens were sent for culture and sensitivity. All blood and sputum results were negative and the urine culture obtained was positive for $>10^{5} \mathrm{CFU} / \mathrm{mL}$ Candida albicans. All other investigations were noncontributory.

What is your approach for the management of bacteriuria in this patient? (Select all that apply)

$\square$ No specific treatment required for Candida albicans (continue current broadspectrum antibiotic regimen).

$\square$ Repeat urine culture.

$\square$ Change Foley catheter.

Start systemic antifungals for $C$. albicans.

Start local antifungals (i.e., bladder irrigation) for $C$. albicans.

$\square$ Start systemic antifungals based on repeat culture results.

Start local antifungals (i.e., bladder irrigation) based on repeat culture results.

Other. Please specify:

In your view, how likely is this woman to have a urinary tract infection)?

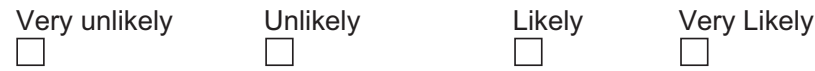


Part 2: Current Practices and Perceptions of Bacteriuria in the Intensive Care Unit (ICU)

Practices

1. Do you use a protocol for the diagnosis and management of urinary tract infection (UTI) in your center?

$\square$ Yes $\square$ No

2. Given a positive urine culture ( $>10^{5}$ colony-forming units $/ \mathrm{mL}$ ) for enteric Gramnegative bacteria, obtained from an indwelling (>48 hrs) Foley catheter in an ICU patient who has been in the ICU for $>48 \mathrm{hrs}$

A. How helpful do you find the following diagnostic tests and/or clinical variables in guiding your management of UTI?

Urine microscopy (e.g., white blood cell count)

$\square$ Very helpful $\square$ Somewhat helpful $\square$ Somewhat unhelpful $\square$ Very unhelpful

Urine biochemistry (e.g., nitrites, leukocyte esterase)

$\square$ Very helpful $\square$ Somewhat helpful $\square$ Somewhat unhelpful $\square$ Very unhelpful

Symptomatology (e.g., dysuria, frequency, urgency)

$\square$ Very helpful $\square$ Somewhat helpful $\square$ Somewhat unhelpful $\square$ Very unhelpful

Urine appearance (e.g., pyuria, color changes, odor, hematuria)

$\square$ Very helpful $\square$ Somewhat helpful $\square$ Somewhat unhelpful $\square$ Very unhelpful

Systemic inflammatory response (e.g., white blood cell count elevation, tachycardia, tachypnea, temperature)

$\square$ Very helpful $\square$ Somewhat helpful $\square$ Somewhat unhelpful $\square$ Very unhelpfu

Hemodynamic status of the patient (e.g., hypotension \pm pressor requirements)

$\square$ Very helpful $\square$ Somewhat helpful $\square$ Somewhat unhelpful $\square$ Very unhelpfu

B. How frequently do you employ the following management approaches in a patient with a positive urine culture for enteric Gram-negative bacteria obtained from an indwelling Foley catheter that has been placed $>48 \mathrm{hrs}$ ?

a. Foley catheter change only

$\square$ Almost never $\square$ Rarely $\square$ Sometimes $\square$ Frequently $\square$ Almost every time

b.Foley catheter change and reculture

$\square$ Almost never $\square$ Rarely $\square$ Sometimes $\square$ Frequently $\square$ Almost every time

c.Treatment with systemic antimicrobials (antibiotics and/or antifungals)

$\square$ Almost never $\square$ Rarely $\square$ Sometimes $\square$ Frequently $\square$ Almost every time

d.Treatment with local antimicrobial administration (e.g., bladder irrigation)

$\square$ Almost never $\square$ Rarely $\square$ Sometimes $\square$ Frequently $\square$ Almost every time

C. What is the level of evidence supporting your treatment approach based on? (Select all that apply)

Randomized controlled studies

Meta-analyses/systematic review

Cohort studies

Case-series reports

Published consensus guidelines

Local practice patterns

Personal experience

Perceptions

1. How often do you encounter a positive urine culture while managing critically ill patients in a typical week in the ICU?

$\square$ Almost never $\square$ Rarely $\square$ Sometimes $\square$ Frequently $\square$ Almost every time

2. How often do you think the following complications occur in an ICU patient as a result of a positive urine culture?

A.UTI (isolated cystitis)

$\square$ Almost never $\square$ Rarely $\square$ Sometimes $\square$ Frequently $\square$ Almost every time

B.Bacteremia/fungemia

$\square$ Almost never $\square$ Rarely $\square$ Sometimes $\square$ Frequently $\square$ Almost every time

C.Death

$\square$ Almost never $\square$ Rarely $\square$ Sometimes $\square$ Frequently $\square$ Almost every time 
Part 3: Demographic Information

1.Describe your practice setting

I. In what type of hospital do you practice? (Select all that apply)

$\square$ University

$\square$ Community

$\square$ Community with university affiliation

II. In what province is the hospital located?

\begin{tabular}{l}
$\square$ Alberta \\
\hline$\square$ British Columbia \\
\hline$\square$ Manitoba \\
\hline$\square$ Newfoundland \\
\hline New Brunswick \\
\hline$\square$ Northwest Territories \\
\hline$\square$ Nova Scotia \\
\hline Nunavut \\
\hline$\square$ Ontario \\
\hline$\square$ Prince Edward Island \\
\hline Quebec \\
\hline Saskatchewan \\
$\square$ Yukon
\end{tabular}

2. The next three questions relate to the type of unit in which you practice.

A. How many staffed beds are in the intensive care/ critical care units in which you practice most often?

B. What type of unit is this?

$\square$ Open (any attending physician with hospital admitting privileges can be the physician of record and direct ICU care)

$\square$ “Closed” (only an intensivist is the physician of record for all ICU patients)

C. What is the primary patient population of the unit to which you practice most often? (Check all that apply)

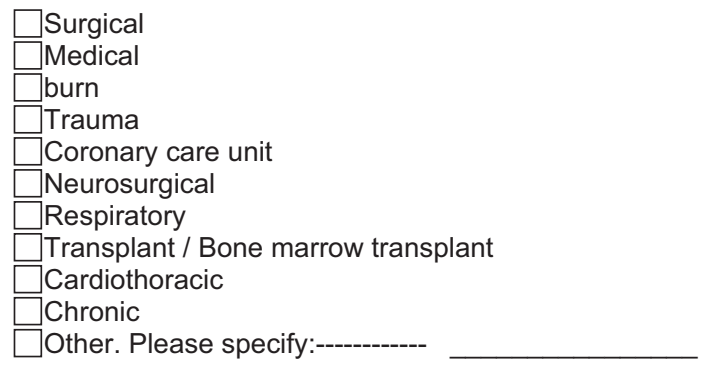

3. Describe your experience as an intensivist.

A. How many years have you been practicing in the ICU?

B. Do you have specific graduate/fellowship training (i.e., $\geq 1 \mathrm{yr}$ ) in intensive care medicine?

$\square$ Yes $\quad \square$ No

If yes, how many years?

C. What is your base specialty? (Check all that apply)

$\square$ Respirology

$\square$ Anesthesiology

$\square$ Surgery

Trauma

$\square$ Emergency medicine

Internal medicine

$\square$ Other. Please specify:

D. What percent of your clinical or total time (i.e., compared with other activities like research or teaching) is spent managing critically care patients?

$\square 0-20 \% \square 21-40 \% \square 41-60 \% \quad \square 61-80 \% \quad \square 81-100 \%$

Thank you for completing the survey 